\title{
A importância da relação médico-paciente para o tratamento oncológico: uma revisão bibliográfica
}

\author{
The importance of the doctor-patient relationship for cancer treatment: a review \\ bibliographic
}

La importancia de la relación médico-paciente para el tratamiento oncológico: uma revisión bibliográfica

\begin{abstract}
Elissa de Carvalho Portela ${ }^{1 *}$, Igor Azevedo Caldas ${ }^{1}$, Isabella Araújo Ribeiro, Sávio Régis Mesquita Pinheiro ${ }^{1}$, Vinícius Diego Mendes Silva ${ }^{1}$, Renata Paula Lima Beltrão ${ }^{1}$, Augusto Cesar Beltrão da Silva ${ }^{1}$.
\end{abstract}

\section{RESUMO}

Objetivo: O objetivo desta revisão é verificar de que maneira a relação médico-paciente pode interferir positivamente no processo de tratamento de pacientes com câncer. Revisão bibliográfica: $O$ câncer se tornou uma das patologias mais temíveis na sociedade atual. As singularidades que exige o seu tratamento consideram a forte necessidade de uma abordagem biopsicossocial do problema e de um cuidado humanizado em direção a pacientes e familiares, uma vez que ocasiona desordens psicológicas e emocionais que afetam, inclusive, a vivência de qualidade de vida dos acometidos pela doença. A patologia do câncer, propriamente dita, resulta em dor e desconforto, assim como seu tratamento químico, que amplia a fragilidade dos pacientes. Além disso, a rotina de tratamento torna-se exaustiva, à medida que implica a necessidade de um retorno frequente ao ambiente hospitalar e na exposição a medicamentos que provocam a lassidão do paciente. Considerações finais: A relação médico-paciente se faz enquanto aspecto crucial para o tratamento de pacientes oncológicos, tendo em vista que permite aos acometidos melhores formas de lidar com a doença e com a vida em sociedade para além do hospital, promovendo o bem-estar desses indivíduos.

Palavras-chave: Câncer, Relação médico-paciente, Tratamento humanizado.

\begin{abstract}
Objective: The objective of this review is to verify how the doctor-patient relationship can positively interfere in the treatment process of cancer patients. Bibliographic review: Cancer has become one of the most fearsome pathologies in today's society. The singularities that require its treatment consider the strong need for a biopsychosocial approach to the problem and a humanized care towards patients and family, since it causes psychological and emotional disorders that affect, including the experience of quality of life of those affected by the disease. The pathology of cancer itself results in pain and discomfort, as well as its chemical treatment, which amplifies the frailty of patients. In addition, the routine treatment becomes exhaustive as it implies the need for a frequent return to the hospital environment and exposure to medicines that cause the patient's lassitude. Final considerations: The doctor-patient relationship is a crucial aspect for the treatment of cancer patients, given that it allows the affected people better ways to deal with the disease and with life in society beyond the hospital, promoting the well-being of these individuals.
\end{abstract}

Keywords: Cancer, Physician-patient relationship, Humanized treatment.

${ }^{1}$ Faculdade de Ciências Humanas, Exatas e da Saúde do Piauí (FAHESP), Parnaíba - PI.

*E-mail: viniciussdiego@gmail.com

SUBMETIDO EM: 11/2020

ACEITO EM: 12/2020

PUBLICADO EM: $3 / 2021$ 


\section{RESUMEN}

Objetivo: El objetivo de esta revisión es verificar de qué manera la relación médico-paciente puede interferir positivamente en el proceso de tratamiento de pacientes con cáncer. Revisión bibliográfica: El cáncer se ha convertido en una de las patologías más temibles en la sociedad actual. Las singularidades que exige su tratamiento consideran la fuerte necesidad de un abordaje biopsicosocial del problema y de un cuidado humanizado en dirección a pacientes y familiares, ya que ocasiona desordenes psicológicos y emocionales que afectan, incluso la vivencia de calidad de vida de los afectados por la enfermedad. La patología del cáncer, propiamente dicha, resulta en dolor y malestar, así como su tratamiento químico, que amplía la fragilidad de los pacientes. Además, la rutina del tratamiento se hace exhaustiva, ya que implica la necesidad de un retorno frecuente al entorno hospitalario y la exposición a medicamentos que provocan el agotamiento del paciente. Consideraciones finales: La relación médico-paciente es un aspecto crucial para el tratamiento de los pacientes oncológicos, dado que permite a las personas afectadas afrontar mejor la enfermedad y con la sociedad además del hospital, promoviendo el bienestar de estas personas.

Palabras clave: Cáncer, Relación médico-paciente, Tratamiento humanizado.

\section{INTRODUÇÃO}

O câncer é compreendido na atualidade como o maior problema de saúde pública do mundo, de acordo com o Instituto Nacional do Câncer (INCA) (2019), ele se constitui enquanto um dos principais causadores da morte prematura no Brasil e no mundo, e os índices de acometimento da doença são cada vez maiores. Seu diagnóstico é, geralmente, acolhido pelos pacientes com sentimento de insegurança e medo, sobretudo, por se tratar de uma patologia cujo tratamento é invasivo e pungente.

O INCA (2019) prevê que os anos de 2021 e 2022 incorporarão uma enorme alta nos casos de câncer no Brasil, dos quais o câncer de pele não melanoma é o mais frequente, acompanhado pelos cânceres de mama, próstata, colón e reto, respectivamente.

O anúncio do diagnóstico de uma doença estabelece uma divisão entre o estado anterior de confiança do paciente em si, e a capacidade de afrontar o mundo. No modo que está segurança em um estado de desilusão após a perda da ilusão de que nada poderia acontecer de mortal. Esta desilusão se traduz pela fala frequentes desses pacientes: Eu nunca pensei que realmente um dia isto poderia acontecer comigo (MARTIN CLS, 2006).

O estudo a respeito da relação médico-paciente tem se tornado uma temática cada vez mais debatida no meio científico e de pesquisas relacionadas ao tratamento de doenças. De modo que este recorte se posicionou enquanto crucial à formação nos cursos voltados à área da saúde, procurando formar profissionais capazes de realizar uma intervenção que acompanhe a doença por um viés biopsicossocial, promovendo melhores condições de tratamento aos pacientes no ambiente hospitalar, ao passo que este disponha de qualidade de vida, também, em sociedade (GERRERO ME, et al., 2011).

Ao longo dos anos as concepções a respeito do conceito de saúde foram sendo remoldadas. Embora por muito tempo a ideia de saúde tenha se mantido apegada à fatores biológicos, sua abordagem atual encontrase relacionada, também, a outros requisitos, estando inclusas neste cenário as questões psicológicas e sociais (BRENTANI MM, et al., 2003). A partir desta prerrogativa se torna possível a verificação da importância da relação médico-paciente para a intervenção eficaz no tratamento de pacientes oncológicos, haja vista que, a promoção da saúde um aspecto estritamente biológico que deve ser tratado apenas com embasamento técnico.

A espiritualidade e a fé ajudam e influenciam bastante no processo de tratamento do paciente oncológico, pois o próprio paciente pode se ajudar, atribuindo um significado no seu processo de cura, em busca da sobrevivência. Através da resiliência, o paciente passa a ter força de vontade de viver, passa acreditar que aquilo vai passar, e que as coisas vão melhorar. São fontes de apoio para o tratamento. Os pacientes 
oncológicos passam por muitos períodos de descoberta até o tratamento da doença, após a confirmação do diagnóstico o paciente vê sua vida tomar um rumo diferente. Primeiramente passam por estado de choque, depois percebem que é real, muitas das vezes relutam contra a ideia, mas depois acabam aceitando e planejando um futuro com esperança (GERRERO ME, et al., 2011).

Os cuidados paliativos são aqueles que focam na pessoa e não na doença, que procuram uma melhoria de vida, no paciente oncológico ele afeta não só o paciente como toda a família e até mesmo os profissionais da saúde envolvidos no tratamento. A partir do diagnóstico do câncer e durante o tratamento percebe-se uma ligação muito grande entre os profissionais da saúde e o paciente, minimizando assim o impacto da doença. A humanização, empatia, amparo prestada pela equipe médica durante a realização dos cuidados paliativos influencia diretamente no tratamento da doença, se tornando menos doloroso e mais digno (ANJOS ACY e ZAGO MMF, 2006).

O Apoio emocional é fundamental durante essa luta incessante contra esta doença, pois essa terapia irá desgastar muito o paciente, fazendo com que ele fique bastante debilitado e que comece a sofrer algumas consequências como perda de cabelo durante o tratamento, sobre o emocional do paciente acredita-se que tem como principal responsável o médico (ROSA BM, et al., 2018).

O tratamento humanizado tem sido pauta de muitas discussões entre as ciências da saúde, por meio da qual, através de uma revisão bibliográfica, este trabalho pretende descrever, por meio de uma revisão narrativa sobre os efeitos que a relação médico-paciente possui para os pacientes em tratamento contra 0 câncer.

\section{REVISÃO BIBLIOGRÁFICA}

O câncer, também conhecido como neoplasia maligna, pode ser compreendido como o aumento desenfreado de células, atingindo a órgãos e tecidos. $O$ tratamento baseado apenas em aspectos biológicos e técnicos foi por muito tempo compreendido como suficiente no cuidado tanto de pacientes com câncer, quanto em outros quadros. Todavia, compreende-se a necessidade de tratamentos paliativos neste contexto, visando resultados mais efetivos (LORENCETTI A e SIMONETTI JP, 2005).

Tratar pacientes oncológicos partir desta perspectiva está relacionado a amparar além da fisiologia do paciente, seu estado emocional, e social, como afirma Duncan BB (2014) que classifica as áreas que podem ser acompanhadas neste processo: Tratar da área física diz respeito ao acompanhamento das respostas do corpo do paciente, administrando recursos que melhorem essa condição. Tratar o psicológico está relacionado a perceber e a compreender as necessidades do psíquico daquele paciente, incluindo a verificação de sintomas de estresse, depressão e outros. O cenário social se aplica quando o profissional procura envolver a família nessa dimensão de tratamento, alertando as possibilidades e consequências.

Além dessas dimensões, Duncan BB (2014) também trabalha a explanação da dimensão espiritual, onde o profissional da área da saúde procura compreender os intentos e crenças religiosas do paciente, procurando demonstrar sentimento de empatia. A relação médico-paciente, frente a este processo se faz profundamente necessária, uma vez que ampara as múltiplas necessidades deste segundo.

Embora as prerrogativas envolvidas ao ambiente hospitalar sejam a da promoção e manutenção da saúde, nem sempre este ambiente transmite a sensação de bem-estar aos pacientes, excepcionalmente aqueles com quadros que carecem de internação ou em tratamento constante (é o caso dos acometidos de câncer), cujo retorno é frequente. Diariamente são visualizadas situações de pacientes que se encontram em estados terminais, familiares e acompanhantes com emocional abalado em virtude dessas condições de saúde, e mesmo pessoas evoluindo para óbito estes são alguns dos fatores responsáveis por possíveis entraves ao tratamento de pacientes oncológicos (VEIT MT e CARVALHO VA, 2010).

O trabalho oncológico faz se necessário pois, mas dá metade da população sofre com está comorbidade, o departamento de informática do sistema único de saúde (DATASUS) faz uma espécie de registro em cada local, apresentando uma taxa que passa a casa dos 10 milhões, superando muitos países subdesenvolvidos. 
Algumas localidades são, mas afetadas como no Sudeste $1 / 3$ dos casos do câncer estando registrados nessas cidades, acarretado por problemas Socioeconômicos ligados a muitas vezes a não prática de exercícios físicos e alimentação saudável (BRASIL, 2015).

A perspectiva da humanização é um aspecto visível nas concepções de Duncan BB (2014), e esta é uma das temáticas mais abordadas para o acompanhamento de pacientes no ambiente hospitalar. Pensando este assunto, é interessante posicionar duas vertentes interessantes cujo envolvimento da temática é a relação médico-paciente nas ações do hospital. A primeira é a humanização do tratamento, assunto cada vez mais debatido entre o meio científico e de saúde, e a segunda é sobre as possibilidades para a atuação do médico neste espaço.

Embora a medicina seja um dos recortes profissionais mais antigos do mundo no tocante à área da saúde, de acordo com Carvalho MM (2015) ela é uma das mais atuais. Suas perspectivas de atuação se renovam constantemente, o que, na opinião do autor, inclui suas áreas de abordagem, objetos de estudo e também os espaços onde exerce suas funções, sempre atendendo à uma multiplicidade de variáveis.

A relação no ambiente hospitalar no tocante a estes cuidados paliativos pode atuar de diversas maneiras. O conhecimento do médico sobre os aspectos psicológicos e seus indicadores em direção à condição física é um diferencial que estes profissionais possuem. A ação médica não deve se limitar à relação restrita com o paciente, devendo ser incluída neste processo os familiares (MARISCOTTI J, 2008).

O Paternalismo médico surgiu com significante presença estatística no estudo, sendo comprovando a dificuldade de limitar as tênues fronteiras existentes entre autonomia do paciente e o ato beneficente do médico. Apesar dos profissionais do universo estudado informar certo diagnóstico e prognóstico da doença aos pacientes, este número diminui quando a situação do paciente torna-se terminal; nestes casos, os entrevistados transferem a informação exclusivamente à família. A atitude paternalista emerge em certa medida que os recursos se esgotam. Os médicos têm opiniões contrárias ao relacionar paternalismo e o que é fazer o bem ao paciente, sendo revelado que muitos ainda acreditam na atitude paternalista como forma de fazer o bem (TRINDADE ES, 2007).

Trabalhar a questão da relação médico-paciente para o tratamento de pacientes oncológicos é pensar nos efeitos de todos os fatores possíveis que possam estar diretas ou indiretamente ligados com a determinação propriamente dita dos sintomas da doença, bem como de sua frequência, agravamento ou intensidade. A etiologia do câncer fundamenta-se sob múltiplos fatores, considerando desde à propensão genética do indivíduo, à sua exposição à aspectos considerados de risco (CARVALHO MM, 2015).

Quando se pensa em aspectos biológicos refere-se a fatores como o gênero da doença, a vulnerabilidade genética do paciente, suas funções imunes, os efeitos que as medicações provocam em sua corrente sanguínea, dentre outros aspectos; Enquadrando-se, portanto uma perspectiva psicossocial, pensa-se as atitudes pessoais dos pacientes, sua personalidade, emoções traumáticas, o suporte que o paciente possui em sociedade, seu histórico familiar, educação, seu trabalho e ocupações, além de suas culturas e perfil econômico. De modo que, está multiplicidade de fatores possa gerar disfunções no organismo, dificultando seu tratamento (ANJOS ACY e ZAGO MMF, 2006).

Cabe dizer ainda que um aspecto importante nessa relação médico-paciente no que se refere a casos oncológicos trata-se da autonomia do paciente. Isso implica dizer que o paciente é o dono do seu próprio destino, tendo em vista que as suas decisões devem ser ouvidas na relação médico-paciente. Com isso se faz necessária uma comunicação adequada e ativa nessa relação, devendo o médico usar a razoabilidade para comunicar a situação clínica do paciente. Isso implica na quebra da ideia que predominava há décadas atrás, de que o paciente não deveria ter conhecimento do seu diagnóstico, para que o seu sofrimento fosse amenizado (MORAIS GB, et al., 2018).

\section{As etapas do estabelecimento da relação médico-paciente em pacientes oncológicos}

Segundo Brentani MM, et al. (2003), o primeiro passo para o estabelecimento de um tratamento em que se preze por uma boa relação médico-paciente é a visualização classificativa deste paciente pelo olhar de 
seu médico, ou seja, a classificação do paciente, diga-se sobre os estágios da doença e impactos que ela gera biopsicossocialmente para o indivíduo. Esta classificação inclui, ainda, a verificação do histórico médico do paciente, identificando a possibilidade da relação com fatores patológicos anteriores ou elementos biopsicossociais que tenham favorecido a condição em que se encontra.

A próxima etapa deste processo está relacionada ao diagnóstico que, de acordo com Brentani MM, et al. (2003), não pode ser determinado como uma via de regra universal. Para o autor, os diagnósticos variam de acordo com a relação sintomática dos pacientes. Dado o diagnóstico, inicia-se um processo mais rigoroso de acompanhamento. Nesta etapa o diálogo com o paciente é imprescindível, visto que permite o conhecimento das singularidades a serem enfrentadas posteriormente, neste sentido é que podem ser trabalhadas algumas técnicas para uma melhor receptividade do paciente ao tratamento, permitindo-lhe a apreensão das informações sobre a doença a ser tratada e das possibilidades que ele tem a partir deste enredo.

No caso específico de consultas em oncologia, por exemplo, grande parte das dificuldades está funcionalmente relacionada ao contexto de comunicação de más notícias, no qual médicos enfrentam, continuamente, a necessidade de transmitir a pacientes e familiares informações complexas, capazes de alterar drasticamente suas perspectivas de futuro e, consequentemente, geradoras de grande ansiedade (BAILE WF e AARON J, 2005).

É fundamental que o paciente esteja sempre bastante informado sobre sua situação, para que assim ele estando envolvido no seu tratamento mantenha mais esperanças de uma possível evolução no estado clínico, por isso é muito importante que haja sempre uma boa comunicação entre o profissional e o paciente na área de saúde, principalmente quando nos referimos à oncologia (ZABOLI ELCP e SARTORIO NA, 2006).

Quanto à necessidade de manutenção da esperança do paciente, motivo alegado por profissionais e famílias para omitir informações daquele que estava adoecido e incentivar percepções e expectativas irreais sobre sua condição, o que na nossa análise aponta é que, em se tratando de "falsa esperança. Os primeiros modelos de comunicação, na primeira metade do século XX, surgem justamente da constatação da possível divergência entre aquilo que o emissor de determinada mensagem pretende comunicar e o que efetivamente chega ao receptor (ZABOLI ELCP e SARTORIO NA, 2006).

$\mathrm{Na}$ escolha das palavras-estímulo, sempre tentei utilizar o maior número possível de palavras correntes na linguagem coloquial, de modo a evitar dificuldades de intelecção. Contudo, esse não é o caso. Nas palavras mais simples surgem hesitações ou outros distúrbios que podem apenas ser explicados pelo fato de que um complexo foi acionado com a palavra estímulo (JUNG CG, 2007).

Nesta fase, é necessário que o médico responsável ou a equipe se disponha a dialogar com o paciente, e, se possível, incluir no tratamento, profissionais capacitados para melhor compreender as inquietações deste indivíduo, sendo importante considerar a necessidade de realização de aplicação de questionários para avaliação futura de suas respostas e analise da condição psicológica e social ao qual está inserido (CARVALHO MM, 2002).

É importante relatar que cada profissional da saúde tenha a devida integridade no tratamento da sua enfermidade tendo autonomia nas suas decisões priorizando sempre seu paciente como afirma a política Nacional de Humanização (PNH). Fazendo com que a família seja um componente essencial nas possíveis tomadas de decisão entre médico e paciente mostrando os prós e contras de um tratamento voltado para as pessoas que sofrem de câncer, incentivando a educação em saúde (TADDEO PS, et al., 2012)

A definição do plano de tratamento só pode ser traçada mediante a realização de todas estas etapas, e as estratégias de intervenção devem pensar as respostas do paciente, assim como a interferência de sua inserção em família e sociedade, além de suas condições psicológicas individuais, como o estresse e indícios de depressão (VEIT MT e CARVALHO VA, 2010).

Para o estabelecimento de uma boa relação médico-paciente é necessário, portanto, que o médico procure identificar a maneira com a qual o paciente interpreta o seu problema de modo interno e percebendo a preexistência de problemas psicológicos-comportamentais que interajam no seu tratamento, tornando possível a integração de equipe multidisciplinar para melhor atender ao quadro. Além dessa função, a relação 
médico-paciente pode ser crucial para possibilitar ao portador de câncer o entendimento da necessidade de dar prosseguimento aos modelos de tratamentos ao mesmo tempo em que este pode levar uma vida normal para além do hospital (ANJOS ACY e ZAGO MMF, 2006).

Embora seja crucial a boa relação médico-paciente independentemente da patologia, os pacientes oncológicos são destaque por uma série de fatores. O diagnóstico da doença acarreta crises emocionais e existenciais, e o avançar da doença e intervenção química provocam modificações no corpo que, nem sempre, são compreendidas e/ou superadas pelos pacientes. Em sua maioria, os pacientes oncológicos têm perda significativa de peso, além de dores constantes, fraqueza, além de questões estéticas afetadas pela matéria prima da medicação utilizada. A queda dos pelos é uma das características mais conhecidas pelo público em geral (DUNCAN BB, 2014).

Considerando as dificuldades do custeio em medicamentos e no modo de vida operante por cada paciente se pode notar as grandes diferenças Socioeconômicas da população, muitos tem receio da doença as vezes ligado a falta de informações causado pela alta letalidade dessa doença com incidência maior de casos por linfoma não-Hodgkin 2,4\% seguidos de câncer no sistema Nervoso Central 2,3 \% e outras neoplasias, ligados a fatores hereditários ,tabagismo, obesidade hormonal e ao envelhecimento (ZABOLI ELCP e SARTORI NA, 2006)

A boa relação médico-paciente permite, portanto, a verificação da maneira que este paciente se relaciona com as questões mencionadas acima, e que variáveis possam estar provocando surgimento, manutenção e/ou agravamento do quadro (TRINDADE ES, et al., 2007). Neste sentido, o médico deve procurar remodelar os critérios que subsidiam o tratamento, proporcionando maior qualidade ao acompanhamento deste indivíduo, mostrando ao paciente suas possibilidades de lidar com estes problemas.

É notório salientar sobre o eixo de temática médico e paciente, seja um tratamento na qual o paciente esteja totalmente seguro, e as consequências desse tipo de solução, o diagnóstico em fase inicial da doença passando por quimioterapia e radioterapia na finalidade de melhorar a sobrevida de cada pessoa. Normalmente esse tipo de tratamento e algo muito exaustivo que vai gerar alguns transtornos para a vida de seus pacientes e familiares. Dialogar e se disponibilizar a ouvir as queixas pode ser um dos principais elementos para um cuidado mais efetivo quando se trata de neoplasias (SORATTO MT e SILVA DM, 2016).

A equipe de enfermagem que atua em oncologia precisa desenvolver habilidades, tais como ter cuidado e interesse pelo outro, dar-se, estar aberto para discutir a fé, encorajar a esperança, escutar atentamente, demonstrar confiança e honestidade, orar a pedido da família ou do paciente, tocar a pessoa, dar abertura, ajudar o paciente no encerramento das questões do passado e amar (PIMENTA AG, et al., 2006).

A ideia da humanização dentro do espaço hospitalar é um dos principais diferenciais que traz a perspectiva da relação médico-paciente, funcionando como crucial para a transformação da conduta médica junto aos pacientes. A técnica foi por um longo período uma das únicas preocupações das equipes médicas e esta transformação vem para agregar a esta ação, considerando a pessoalidade desse processo. Isto implica saber do paciente sobre suas inseguranças, angústias e medos, quer sejam eles relacionados ao próprio ambiente hospitalar, problema de saúde, ou procedimento de tratamento ao qual irá se submeter, ou não, podendo se estender a questões externas que possam interferir negativamente para a manutenção de sua saúde (DUNCAN BB, 2014).

\section{CONSIDERAÇÕES FINAIS}

Desde o diagnóstico à vivência com o tratamento contra o câncer os pacientes são tomados por uma série de conflitos internos, existenciais, sobretudo por ser um dos problemas que mais provocam o óbito no campo da saúde atualmente. $O$ desprendimento aos conceitos de tratamento estritamente técnico e de um relacionamento imparcial com os pacientes tem sido uma prática questionável no meio científico atual, que tem procurado melhores estratégias de tratamento aos pacientes pautadas no processo de humanização, que tem se mostrado como excelente recurso para uma intervenção médica efetiva, razão pela qual a relação médico-paciente se tornou importante ao tratamento de pacientes com câncer. 


\section{REFERÊNCIAS}

1. ANJOS ACY, ZAGO MMF. A experiência da terapêutica quimioterápica oncológica na visão do paciente. Rev. LatinoAm. Enfermagem, 2006.

2. ARRAIS RH, et al. A vivencia psicológica da comunicação sobre diagnostico e tratamentos por pacientes oncológicos: uma perspectiva da psicologia analítica. Rev. SBPH. 2015; 18: 2.

3. BAILE WF, AARON J. Patient-physician communication in oncology: Past, present, and future. Current Opinion in Oncology., 2005; 17(4): 331-335.

4. BRENTANI MM, et al. Bases da Oncologia. São Paulo: Lemar Livraria Editora Marina e Tecmedd Editora, 2003.

5. CARVALHO MM. Psico-oncologia: História, características e desafios. São Paulo: Psicologia USP, 2002.

6. DUNCAN BB, SCHIMDT MI. Medicina Ambulatorial: Condutas de Atenção Primária Baseadas em Evidência. Porto Alegre: Artmed, 2014

7. INCA. INSTITUTO NACIONAL DE CÂNCER. Estimativa 2020: incidência de câncer no Brasil. Rio de Janeiro: INCA; 2019.

8. LORENCETTI A, SIMONETTI JP. As estratégias de enfrentamento de pacientes durante o tratamento de Radioterapia. Latinoam Enferm., 2005; 13(6): 944-50.

9. MARISCOTTI J. A delicate dance: negotiating the doctor-patient relationship during cancer treatment. Oncologist., 2008; 13(11): 1205-6.

10. MARTIN CLS. O paradoxo do diagnóstico precoce de câncer. Revista latinoam. psicopatol. Fundam, 2006 ; 9 : e3 .

11. MORAIS GB, et al. A Valia do Vínculo na Relação Equipe Multidisciplinar-Paciente Oncológico para a Continuidade do Cuidado: uma Revisão Integrativa. Revista Saúde \& Ciência online, 2018; 7(2): 502.

12. OSANAI MH. Relação médico-paciente em oncologia. CMI Brasil, 2004; 15: e2214.

13. ROSA BM, et al. Comportamento comunicativo em oncologia: perspectiva de médicos e pacientes. Revista Interdisciplinar de Promoção da Saúde, 2018; 1: e 2.

14. SANTOS ALN, LIRA SS. A comunicação médica com pacientes oncológicos frente às barreiras ético-médicas. Dê Ciência em Foco, 2018; 4: e24.

15. SORATTO MT, SILVA DM. A temática médico e paciente nos casos oncológicos. Saúde e Pesquisa, 2016 ; 1 : e32.

16. TADDEO PS, et al. Acesso, prática educativa e empoderamento de pacientes com doenças crônicas. Ciência \& Saúde Coletiva, 2012; 17: e2913

17. TRINDADE ES, et al. O médico frente ao diagnóstico e prognóstico do câncer avançado. Rev. Assoc. Med. Bras, 2007; 1: e53.

18. VEIT MT, CARVALHO VA. Psico-Oncologia: um novo olhar para o câncer. O mundo da saúde, 2010; 34.

19. ZABOLI ELCP, SARTORIO NA. Bioética e enfermagem: uma interface no cuidado. O mundo da saúde, $2006 ; 3:$ e382. 